\title{
COMPARAÇÃO DAS IMAGENS LANDSAT 8 E SENTINEL 2A PARA O MONITORAMENTO DE RESERVATÓRIOS DE ABASTECIMENTO NO CERRADO BRASILEIRO
}

\section{LANDSAT 8 AND SENTINEL 2 IMAGES COMPARISON FOR THE RESERVOIRS SUPPLY MONITORING IN THE BRAZILIAN CERRADO}

\author{
Carolina MELO ${ }^{1}$, Marcio Augusto Reolon SCHMIDT ${ }^{1}$, Rodrigo Mikosz GONÇALVES ${ }^{2}$ \\ ${ }^{1}$ Universidade Federal de Uberlândia, Faculdade de Engenharia Civil Programa de Pós-Graduação em Engenharia Civil. Av. João \\ Naves de Ávila, 2121 - Santa Mônica, Uberlândia - MG. E-mail: cmelo.geo@clarivi.com.br; marcioarschmidt@gmail.com \\ ${ }^{2}$ Universidade Federal do Pernambuco, Departamento de Engenharia Cartográfica. E-mail: rodrigo.mikosz@ufpe.br

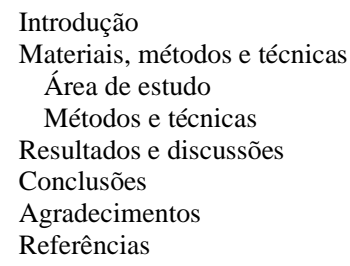

RESUMO - O uso de imagens de satélite como ferramenta de monitoramento ambiental tem grande aplicabilidade em diversas áreas. Contudo, ainda é pouco explorada para o acompanhamento de recursos hídricos devido à falta de parâmetros que correlacionem a qualidade da água e as respostas espectrais. Esta pesquisa avalia o uso das imagens de satélites LandSat 8 e Sentinel 2 para o monitoramento dos parâmetros de turbidez e sólidos totais. O estudo de caso foi realizado para os dois reservatórios de abastecimento do Complexo Capim Branco em Uberlândia, MG, Brasil. As amostras coletadas em campo foram correlacionadas às respostas espectrais nas bandas visíveis e infravermelho próximo e aos índices de Razão Simples com uso do infravermelho próximo (NIR) e Red Edge. Os resultados apresentaram correlação positiva ao aplicar as bandas isoladamente do sensor MSI do satélite Sentinel 2. Para a aplicação dos índices foi observada que as imagens oriundas do LandSat 8 têm maior correlação $\left(\mathrm{R}^{2}>0,8\right)$ considerando polinômios com diferentes graus. Dessa forma os resultados mostraram que o sensor OLI possui maior potencial para a determinação da correlação da turbidez e sólidos totais em reservatórios de abastecimento de médio porte. Destaca-se a importância de validar a resposta espectral para o monitoramento ostensivo de reservatórios e corpos hídricos e assim aprimorar seu uso como complemento às análises convencionais.

Palavras-chave: Radiação eletromagnética. Reservatório. Monitoramento; Sensoriamento remoto.

\begin{abstract}
The satellite images usage as an environmental monitoring tool has great applicability in several areas. However, still little unexplored for water resources monitoring due to the lack of parameters that correlate water quality and spectral responses. This research evaluates the use of LandSat 8 and Sentinel 2A satellites images for turbidity and total solids parameters monitoring. The case study is carried out for the two supply reservoirs of the Capim Branco Complex in Uberlândia, MG, Brazil. The in-situ samples were correlated to the spectral responses in the visible and near infrared bands and to the Simple Reason indices using near infrared (NIR) and borderline (Red Edge). The results showed a positive correlation when applying the bands separately from the Sentinel 2 MSI sensor. For the indices application, it was observed that the images from LandSat 8 have a higher correlation $\left(\mathrm{R}^{2}>0.8\right)$ considering polynomials with different degrees. Thus, the results showed that the OLI sensor has greater potential for determining the correlation of turbidity and total solids in medium-sized supply reservoirs. It is highlighted the importance of validating the spectral response for the reservoirs and water bodies ostensible monitoring, thus enhancing their use as a complement to conventional analyzes.
\end{abstract}

Keywords: Electromagnetic radiation. Reservoir. Monitoring. Remote Sensing.

\section{INTRODUÇÃO}

O sensoriamento remoto vem sendo aplicado no monitoramento de recursos hídricos como forma de reduzir os custos, suprindo as grandes lacunas de espaço e tempo em território brasileiro. Espinoza et al. (2012); Robert et al. (2016) e JafarSidik et al. (2017) afirmam que os métodos comvencionais, que tomam poucas amostras e em geral em pontes ou próximos a bordas dos reservatórios, não conseguem apresentar resultados compatíveis com a necessidade do monitoramento dessas áreas em custos viáveis ao longo do tempo, em especial naqueles corpos hídricos de grande extensão.

O monitoramento por imagens de satélite se destaca no contexto dos reservatórios de abastecimento, pois a redução da capacidade de acumulação de sedimentos carreados não pode ser claramente percebida com um número de amostras não significativas para descrever o reservatório como um todo ou mal distribuídas ao longo do reservatório. Contudo, as variações de turbidez e sedimentos podem ser detectáveis nas imagens obtidas pelos sensores de imageamento orbitais. 
O carreamento de sedimentos tem como principais consequências a redução da vida útil do reservatório pelo assoreamento, afeta a sustentabilidade das estruturas hidrelétricas e o suprimento de água nas estações de captação (Robert et al., 2016).

A baixa velocidade das águas em reservatórios deste tipo afeta também a capacidade de autodepuração, que corresponde ao processo natural de degradação de poluentes, resultando em desequilíbrio físico-químico da água e que pode levar a um processo de eutrofização (Novo et al., 2007). Portanto, a consolidação e manutenção dos reservatórios exigem monitoramento constante para a precaução ou amenização dos problemas comuns, tais como: colapso de barragens, poluição das águas, acompanhamento de vazão e níveis dos reservatórios, quantidade de poluentes e de materiais dissolvidos.

Jafar-Sidik et al. (2017) relatam que os métodos de medições dos parâmetros da água são caros, tanto em tempo quanto em custo, quando comparado com as técnicas de sensoriamento remoto, principalmente em grandes áreas. Contudo, os estudos das águas interiores são opticamente complexas devido à alta concentração de biomassa, partículas minerais, detritos e são altamente variáveis, além de demandarem alta resolução espacial dos sensores remotos (Palmer et al., 2015). Diante desse contexto, vários autores têm estudado modelos para medir os parâmetros da água por sensoriamento remoto (Alcântara et al., 2016; Robert et al., 2016; Woźniak et al., 2016; Abdelmalik, 2018).

Alguns estudos foram realizados utilizando sensores de baixa resolução espacial, tais como Espinoza et al. (2012) e Robert et al. (2016). Ambos utilizaram o sensor MODIS, no entanto as aplicabilidades desses estudos se limitam a áreas extensas, pois estes sensores apresentam baixa resolução espacial. Entretanto, com melhora da resolução espacial dos sensores orbitais e a facilidade de aquisição das imagens, como no caso do satélite LandSat 8 e Sentinel 2A, os estudos nessa temática passam a ser viáveis em áreas menores, tais como pequenos lagos, rios e reservatórios (El-Zeiny \& El-Kafrawy, 2017; Nichol \& Nazeer, 2016; Zhang et al., 2017). Entre as vantagens destes novos sistemas destaca-se a possibilidade do monitoramento em escalas com mais detalhes e diferentes resoluções espectrais.
Exemplos de pesquisas neste contexto são os trabalhos de Espinoza et al. (2012) e Alcântara et al. (2016). Espinoza et al. (2012), que utilizaram as bandas 1 e 2 do sensor MODIS para correlacionar a refletância de pontos ao longo do Rio Madeira com sedimentos em suspensão. Contudo, dada a resolução espacial do sensor, de $250 \mathrm{~m}$, a metodologia dos autores não se torna aplicável em regiões menores, como, por exemplo, o cerrado mineiro, que apresenta cursos d’água de pequeno porte. Alcântara et al. (2016) estimaram o total de matéria em suspensão na água pelo sensor OLI do satélite LandSat 8 em um reservatório de acumulação. Os resultados indicaram que as bandas do azul e verde têm maior capacidade de indicar a presença de matéria orgânica dissolvida quando comparado com as demais bandas deste satélite. Bem como, a presença de grandes quantidades de sólidos dissolvidos e em suspensão e a consequente alteração da turbidez, pode ser detectada através da razão das frequências do vermelho e infravermelho do espectro eletromagnético, corroborando com Espinoza et al. (2012); Robert, et al. (2016) e ElZeiny \& El-Kafrawy (2017). Destaca-se que a matéria orgânica pode ser identificada através das frequências do azul e verde (Alcântara et al., 2016). Esses autores encontraram uma resposta padrão de cada um dos corpos hídricos estudados relacionando com as características físicas destes a bandas específicas dos satélites, obtendo assim uma assinatura individual da região.

Diante desse contexto, o objetivo geral desta pesquisa foi analisar a alteração da resposta espectral da água em função da análise de seus parâmetros físicos de sólidos totais e turbidez, nos reservatórios Amador Aguiar I e II, na região do Triângulo Mineiro, avaliado a viabilidade da aplicação utilizando os sensores do LandSat 8 e Sentinel 2A, ambos de média resolução espacial. Para esta análise foram avaliadas as faixas do azul, verde, vermelho e infravermelho para o LandSat 8 e ainda a banda Red Edge do Sentinel 2. As imagens foram analisadas separadamente para avaliar a capacidade de detecção de sólidos totais e turbidez. Posteriormente criou-se imagens híbridas a partir da razão de bandas de infravermelho e vermelho. As alterações detectáveis foram submetidas aos testes de Anova e de Turkey para identificar a capacidade de detecção da metodologia. 


\section{MATERIAIS, MÉTODOS E TÉCNICAS}

\section{Área de estudo}

Os reservatórios Amador Aguiar I e II fazem parte do Complexo Capim Branco e foram construídos no ano de 2006, no vale do rio Araguari (Figura 1). Integram ainda o complexo, à montante, os reservatórios de Miranda e Nova Ponte.

A região é de domínio do bioma cerrado, um tipo de savana considerada de grande diversidade (Ab’Saber, 2003), caracterizada por possuir duas estações bem definidas ao longo do ano, sendo o verão chuvoso e o inverno seco. Apesar de apresentar umidade baixa e vegetação preparada para longos períodos de estiagem, no cerrado encontra-se grande parte das nascentes das principais bacias hidrográficas da América do Sul, tais como a do São Francisco, do Paraná, do Tocantins/Araguaia, Rio Xingu, além de ser uma região de captação de água que mantém o aquífero Guarani, importante reserva de água subterrânea. O bioma Cerrado, no qual se encontram as maiores bacias hidrográficas da América do Sul (BRASIL, 2017), é uma região de recarga do aquífero Guarani, maior reserva de água subterrânea da América do Sul. Além disso, esse bioma adquiriu importância econômica dada a produção de grãos e carnes para a exportação. Outro fator importante é o crescimento demográfico e o consequente aumento do consumo.

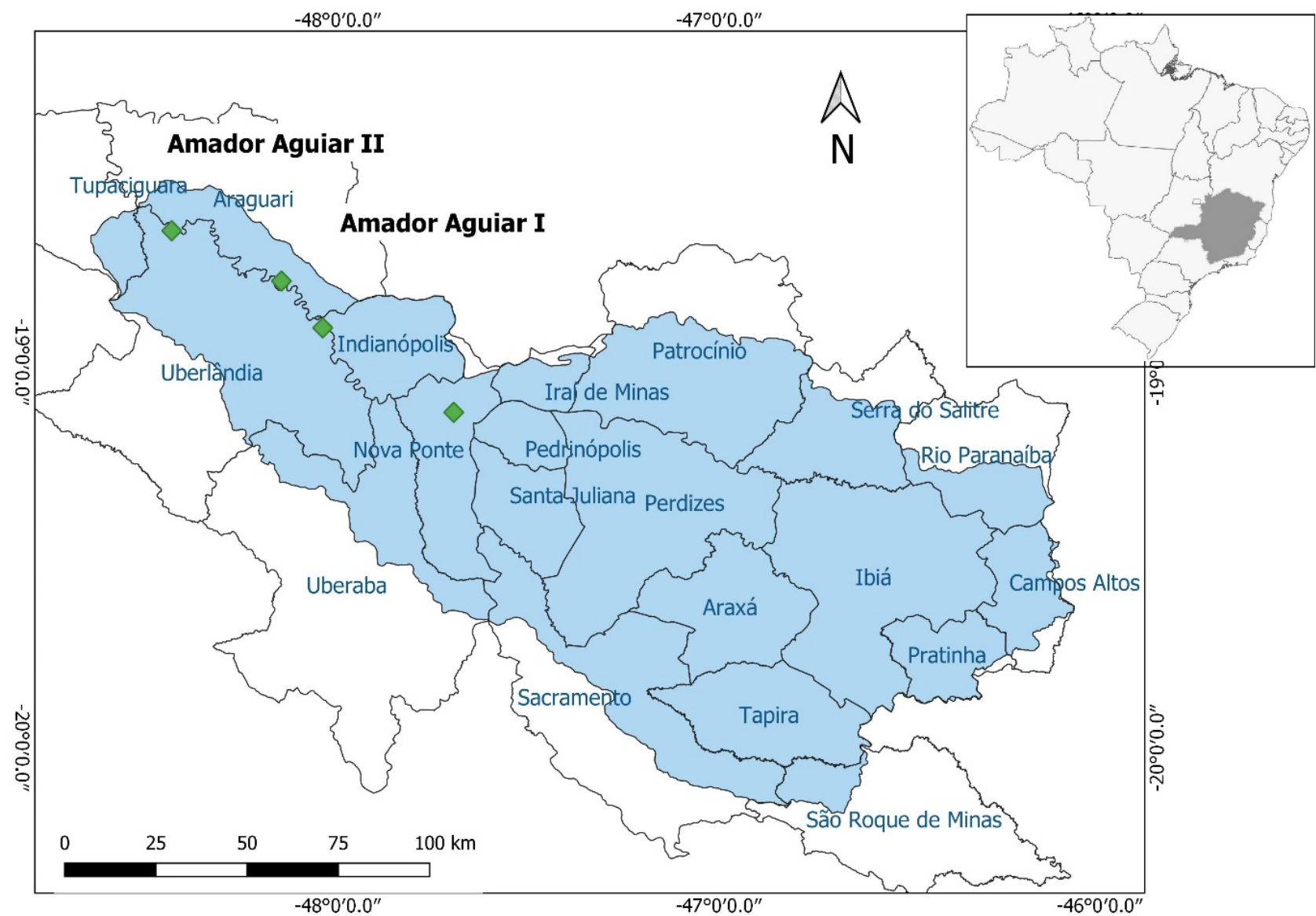

Figura 1 - Área de estudo, reservatórios localizados entre os municípios de Uberlândia e Araguari no Estado de Minas Gerais.

Segundo Souza et al. (2009) a mesorregião do Triângulo Mineiro tem classificação climática de Köppen, como Aw (megatérmico: tropical com verão chuvoso e inverno seco), a estação chuvosa é bem definida no período de outubro a abril e um período seco de maio a setembro. As temperaturas médias ficam entre $14{ }^{\circ} \mathrm{C}$ em Junho e $31{ }^{\circ} \mathrm{C}$ em Dezembro e a precipitação na região oscila entre $1450 \mathrm{~mm}$ e $1750 \mathrm{~mm}$, segundo Novais et al (2018).

O reservatório Amador Aguiar I, segundo o
Consorcio Capim Branco de Energia (CCBE, 2005), possui $18,66 \mathrm{~km}^{2}$ de área inundada, com volume total de armazenamento de 241 milhões de $\mathrm{m}^{3}$, e o reservatório Amador Aguiar II possui área inundada de $45,11 \mathrm{~km}^{2}$, e o volume total corresponde a 872,82 milhões de $\mathrm{m}^{3}$.

\section{Métodos e técnicas}

Foram realizadas coletas de água nos dois reservatórios do complexo Capim Branco, os reservatórios Amador Aguiar I e II, no total de 8 
pontos amostrais distribuídos em três séries em cada reservatório (Figura 2). A coleta no reservatório Amador Aguiar I foi realizada no dia 29 de novembro de 2017 e no reservatório Amador Aguiar II no dia 30 de janeiro de 2018.

As amostras foram coletadas próximas a barragem, devido esse local possuir maior extensão entre as margens, e evitar interferência das margens.

As coletadas foram realizadas à $10 \mathrm{~cm}$ de profundidade, com captura de coordenadas geodésicas utilizando um receptor GNSS (Global Navigation Satellite System) com código C/A e precisão aproximada de 12 metros. Esta precisão é compatível com a resolução espacial dos sensores

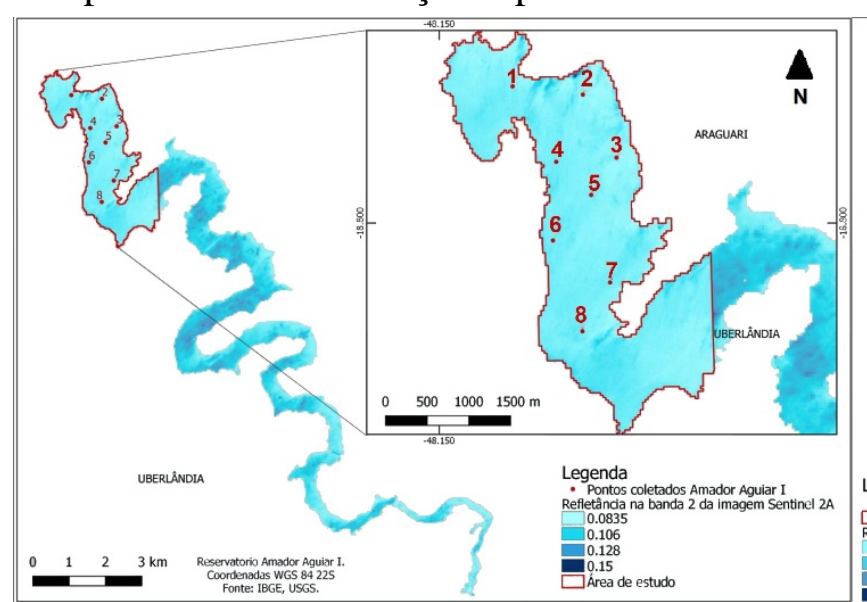

OLI e MSI selecionados, sendo de 30 metros para LandSat 8 e 20 metros para o Sentinel 2A. As amostras coletadas foram armazenadas em garrafas numeradas e com identificação das coordenadas referentes a cada ponto. As leituras de turbidez foram realizadas com a utilização do Turbidímetro AP 2000. Na análise de sólidos totais foram separadas três amostras de cada ponto, contendo $150 \mathrm{ml}$ de água cada, resultando em três análises por ponto a fim de reduzir os erros de medições. Estas foram armazenadas em cápsulas de porcelana previamente calcinadas, pesadas e identificadas. Este material foi colocado em uma estufa para secagem e após esse processo, foi novamente pesado, conforme norma NTS da Sabesp (1999).

Figura 2 - Pontos de coleta das amostras de água nos reservatórios Amador Aguiar I (esquerda) e Amador Aguiar II (direita).

Aos pontos de coleta em cada reservatório foram atribuídos os resultados de sólidos totais e turbidez e exportados para o software Qgis (versão 2.18.2) onde foram espacializados (Figura 2). Neste programa foram avaliados os valores de reflectância dos pixels das imagens selecionadas do LandSat 8 e Sentinel 2A nas coordenadas homólogas com as das amostras e dos oito pixels circundantes.

Os valores presentes nas imagens foram corrigidos dos efeitos atmosfera usando a ferramenta Flaash desenvolvida para corrigir efeitos atmosféricos e correções de azimute de órbita, e possibilitar a compatibilização dos valores obtidos em cada sensor. Essas correções foram aplicadas utilizando os metadados das imagens (data, horário da coleta da imagem, órbita e ângulo solar).

Durante esse processo os pixels das imagens foram transformados para valores de radiância e, posteriormente, para reflectância, que é a representação por porcentagem do nível de energia refletida captada e armazenada em cada pixel.

Foram analisadas as respostas espectrais das bandas do azul, verde e infravermelho próximo (NIR) de ambos os satélites, além da banda Red Edge do Sentinel 2A.

Essas bandas foram selecionadas em função dos estudos de Espinoza et al., (2012 e Robert et al., (2016), que identificaram correlações com sólidos e turbidez no contexto amazônico e sedimentos em suspensão em reservatórios de acumulação no Norte e Nordeste brasileiro usando imagens do sensor MODIS.

A correlação desses parâmetros das amostras coletadas com as respostas espectrais das bandas selecionadas, e com índice de Razão Simples (Rouse et al., 1974) foram utilizadas como por Domínguez et al. (2015), Spitkó et al. (2016) e Souza et al. (2018).

Posteriormente às análises foram determinadas equações polinomiais de cada reservatório, correlacionando turbidez e sólidos totais por meio da correlação $\left(\mathrm{R}^{2}\right)$ conforme abordagens de Espinoza et al. (2012), Robert et al. (2016) e Xie et al. (2018). Por fim, utilizou-se a análise de variância (ANOVA) One Way com o objetivo de verificar se as médias dos valores são 
estatisticamente semelhantes ou não, e posteriormente aplicado o teste de Tukey com nível de significância de 90\%, para verificar quais pontos apresentaram valores diferentes.

\section{RESULTADOS E DISCUSSÃO}

Os resultados de laboratório para sólidos e turbidez apresentaram índices baixos, com pequenas variações em ambos os reservatórios. Devido às características físicas do entorno (geologia, profundidade, vegetação ciliar e localização) serem semelhantes mesmo com a grande dimensão dos reservatórios, não houve diferença entre as amostras.

Isto demonstra uma homogeneidade na qualidade da água do complexo Capim Branco, que engloba os dois reservatórios analisados, na época da coleta. Em vista desta homogeneidade e, a partir do teste da Anova, percebeu-se que duas amostras apresentaram valores discrepantes,

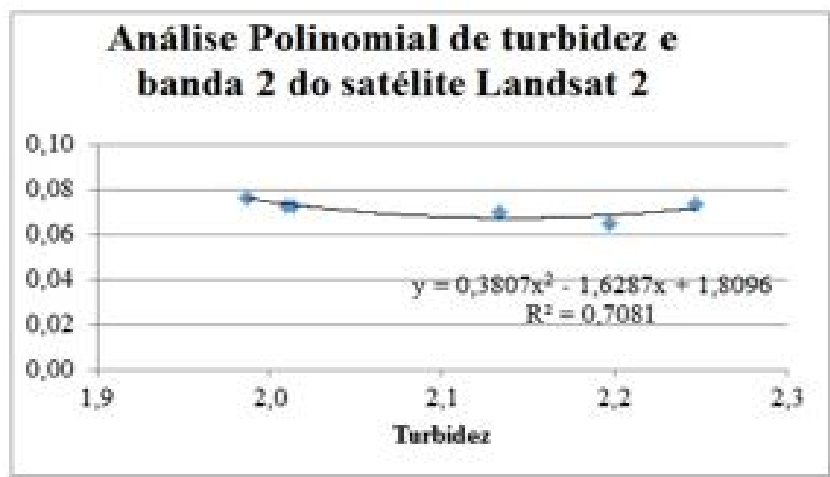

A indicando possivelmente contaminação da amostra, e por não serem espacialmente próximas, foram excluídas da análise.

Na próxima etapa, foi realizada a correlação desses parâmetros das amostras coletadas com as respostas espectrais das bandas diretamente e depois com índice de Razão Simples utilizando as bandas do vermelho, Red Edge $\mathrm{e}$ infravermelho próximo (NIR).

Posteriormente as equações polinomiais de cada reservatório, correlacionando turbidez e sólidos totais, foram determinadas em um gráfico de dispersão (scatter plot) (Figura 3), conforme abordagens citadas anteriormente.

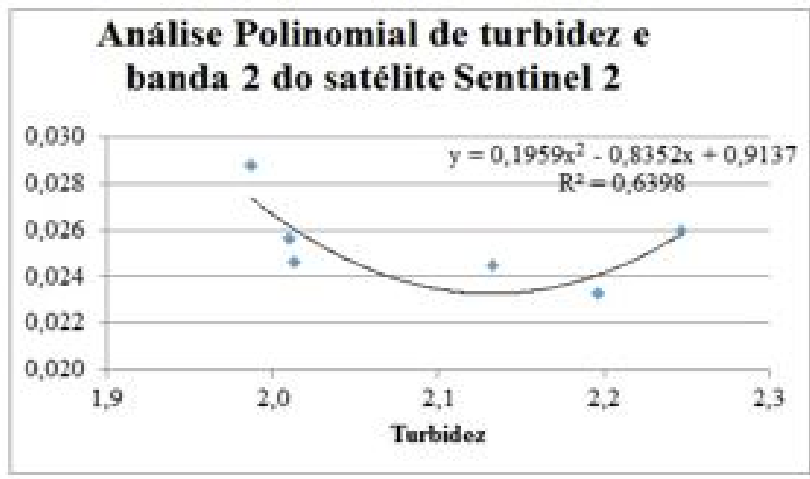

B

Figura 3 - Relação da análise polinomial quadrática dos resultados de turbidez com A) bandas B2 do satélite LandSat 8 e B) banda B2 do satélite Sentinel 2A.

Foram extraídos os valores dos pixels nas coordenadas das amostras de água e os circundantes aos dos pontos amostrais (Figura 4).

No resultado da Anova, dentro das bandas que

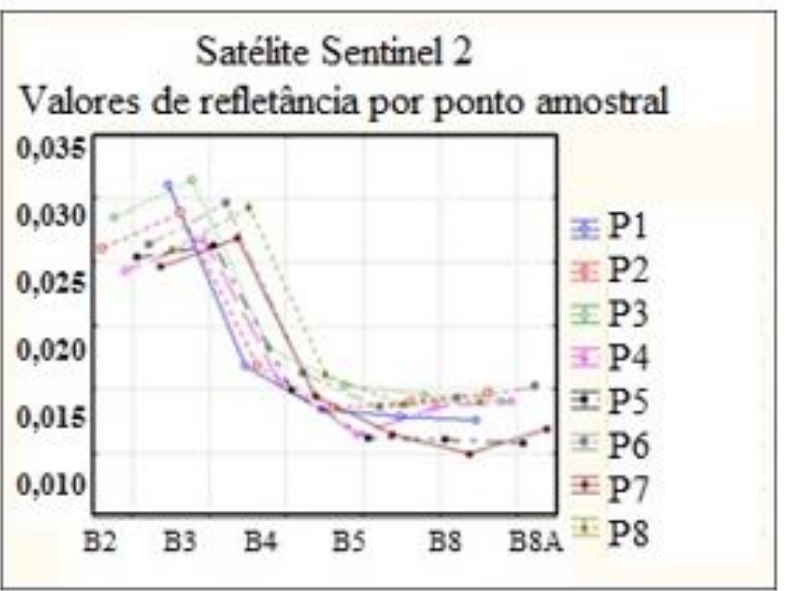

A estão na mesma faixa espectral com os pontos amostrais, não houve diferenças estatisticamente comprovadas ( $\mathrm{p}>0,10$ e $\alpha=0,10)$, quando associado às bandas nos oito pontos amostrais.

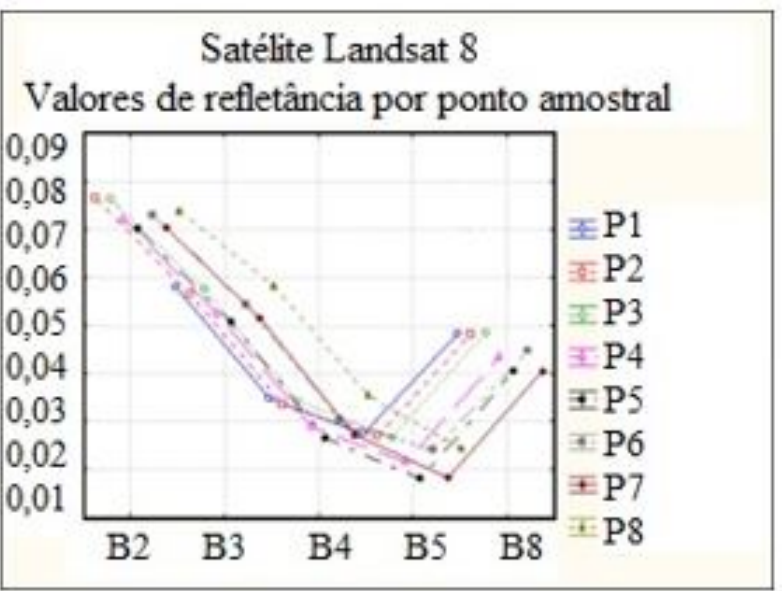

Figura 4 - Variações de respostas da reflectância nas bandas do satélite Sentinel (A) no reservatório Amador Aguiar II e satélite LandSat 8 (B) nos pontos amostrais do reservatório Amador Aguiar II. 
No reservatório Amador Aguiar I a resposta do LandSat 8 foi maior, variando entre 0,11 a 0,18 , enquanto no Sentinel 2 os valores em todos os pontos variaram entre 0,10 a 0,20 . Observa-se também que cada banda no satélite LandSat 8 foi ocupado uma faixa de resposta distinta, enquanto no Sentinel 2A algumas bandas apresentaram faixas de resposta semelhantes, portanto, se confundem.

No reservatório Amador Aguiar II os valores de sólidos totais e turbidez foram maiores do que no Amador Aguiar I, nestes, os valores de resposta nas bandas do azul nos dois satélites foram menores (Figura 4B). No LandSat 8 as respostas em todas das bandas variaram entre 0,02 a 0,08 enquanto no Sentinel 2A a variação foi entre 0,01 a 0,03 (Figura 4A).

As bandas do Azul nos dois satélites obtiveram respostas próximas à banda do verde no reservatório Amador Aguiar II, o que não ocorreu no Amador Aguiar I.

Para obter um modelo que explicasse a relação entre os valores de sólidos totais e turbidez, com os valores resultantes da razão das bandas, foi construído um diagrama de dispersão e ajustados polinômios de diferentes graus, no intuito de verificar em qual tipo de curva e equação do modelo matemático melhor representava a distribuição dos pontos no diagrama. Os resultados estão na Tabela 1. Essa abordagem foi utilizada por Espinoza et al., 2012, Robert et al., 2016 e Xie et al., 2018, para grandes áreas e sensores com baixa resolução.

A aplicação no Cerrado com imagens dos sensores MSI e OLI foi verificar a possiblidade de encontrar uma correlação de forma semelhante.

Tabela 1 - Resultados da análise polinomial ajustada dos parâmetros de sólidos totais e turbidez e as bandas dos satélites LandSat 8 e Sentinel 2A.

\begin{tabular}{|c|c|c|c|c|c|c|c|c|c|}
\hline \multirow[b]{2}{*}{$\begin{array}{l}\text { Ordem do } \\
\text { polinômio }\end{array}$} & \multicolumn{5}{|c|}{ Sentinel 2A, comprimento de onda central (nm) } & \multicolumn{4}{|c|}{$\begin{array}{l}\text { LandSat 8, comprimento de onda } \\
\text { central (nm) }\end{array}$} \\
\hline & $\begin{array}{c}\text { B2 } \\
(490)\end{array}$ & $\begin{array}{c}\text { B3 } \\
(560)\end{array}$ & $\begin{array}{c}\mathrm{B} 4 \\
(665)\end{array}$ & $\begin{array}{c}\text { B8 } \\
(842)\end{array}$ & $\begin{array}{c}\mathrm{B} 8 \mathrm{~A} \\
(865)\end{array}$ & $\begin{array}{c}\text { B2 } \\
(483)\end{array}$ & $\begin{array}{c}\text { B3 } \\
(561)\end{array}$ & $\begin{array}{c}\text { B4 } \\
(654)\end{array}$ & $\begin{array}{c}\mathrm{B} 5 \\
(864)\end{array}$ \\
\hline \multicolumn{10}{|c|}{$\mathrm{R}^{2}$ de Turbidez no reservatório Amador Aguiar I } \\
\hline 2 & 0,1576 & 0,1225 & 0,2075 & 0,2341 & 0,3622 & 0,4468 & 0,4969 & 0,4251 & 0,3904 \\
\hline 3 & 0,2992 & 0,2106 & 0,3202 & 0,261 & 0,4151 & 0,4469 & 0,5797 & 0,4413 & 0,8178 \\
\hline 4 & $\mathbf{0 , 8 8 5 1}$ & 0,943 & 0,3689 & 0,9273 & $\mathbf{0 , 8 3 4 5}$ & 0,4761 & 0,5898 & 0,5374 & 0,8946 \\
\hline 5 & 0,9902 & 0,9989 & 0,7249 & 0,9636 & 0,9222 & 0,674 & 0,5652 & 0,6621 & 0,9722 \\
\hline 6 & 0,676 & $\mathbf{0 , 9 9 7 8}$ & 0,9972 & 0,9948 & 0,9939 & 13,23 & 4,836 & 22,759 & $-6,484$ \\
\hline \multicolumn{10}{|c|}{$\mathrm{R}^{2}$ de Sólidos totais no reservatório Amador Aguiar I } \\
\hline 2 & 0,1841 & 0,2327 & 0,265 & 0,1367 & 0,079 & 0,2818 & 0,149 & 0,127 & 0,3328 \\
\hline 3 & 0,2962 & 0,286 & 0,2956 & 0,2772 & 0,3267 & 0,2989 & 0,3629 & 0,2473 & 0,3829 \\
\hline 4 & 0,3459 & 0,3769 & 0,3887 & 0,2961 & 0,3603 & 0,304 & 0,149 & 0,2502 & 0,9174 \\
\hline 5 & 0,43 & 0,3833 & 0,4074 & 0,3049 & 0,3674 & 0,3898 & 0,3629 & 0,2537 & 0,9186 \\
\hline 6 & 0,6378 & 0,6741 & 0,6394 & 0,5716 & 0,6646 & 0,7414 & 0,9031 & 1,0032 & 0,9962 \\
\hline \multicolumn{10}{|c|}{$\mathrm{R}^{2}$ de Turbidez no reservatório Amador Aguiar II } \\
\hline & B2 & B3 & B4 & B8 & B8A & B2 & B3 & B4 & B5 \\
\hline 2 & 0,6398 & 0,6861 & 0,7178 & 0,8749 & 0,8538 & 0,7081 & 0,8295 & 0,8297 & 0,8512 \\
\hline 3 & 0,7019 & 0,7295 & 0,7795 & 0,9085 & 0,8599 & 0,7488 & 0,8318 & 0,8311 & 0,8546 \\
\hline 4 & 0,9863 & $\mathbf{0 , 9 0 7 1}$ & 0,9299 & 1 & 0,9693 & 1 & 0,9902 & 1 & 0,9969 \\
\hline 5 & 1 & 1 & 1 & 1 & 1 & 1 & 1 & 1 & 1 \\
\hline 6 & 1 & 1 & 1 & 1 & 1 & 1 & 1 & 1 & 1 \\
\hline \multicolumn{10}{|c|}{$\mathrm{R}^{2}$ de Sólidos totais no reservatório Amador Aguiar II } \\
\hline 2 & 0,6607 & 0,5075 & 0,6997 & 0,455 & 0,5298 & 0,3921 & 0,3993 & 0,4157 & 0,4966 \\
\hline 3 & 0,8566 & 0,6652 & 0,7961 & 0,455 & 0,565 & 0,4051 & 0,4217 & 0,4353 & 0,5299 \\
\hline 4 & 0,9014 & 0,6699 & 0,8072 & 0,5203 & 0,6151 & 0,586 & 0,4219 & 0,4387 & 0,5727 \\
\hline 5 & 1 & 1 & 1 & 1 & 1 & 1 & 1 & 1 & 1 \\
\hline 6 & 1 & 1 & 1 & 1 & 1 & 1 & 1 & 1 & 1 \\
\hline
\end{tabular}

No reservatório Amador Aguiar I obteve-se valores de correlação positiva para o parâmetro de turbidez, sendo que o mesmo não ocorreu no Amador Aguiar II. Além disso, valores discrepantes ou mesmo valor 1 indicam a fragilidade do modelo para aplicação das bandas individualmente.

Os valores de $\mathrm{R}^{2}$ obtidos podem sofrer variações locais e climáticas. No teste Anova foi indicada que as variações desses valores podem ser desprezadas, pois são estatisticamente iguais. Os valores de sólidos totais tiveram pouca 
correlação em ambas os reservatórios.

Os valores de refletância maiores nas bandas azul e verde, indicam baixa quantidade de sólidos dissolvidos, assim como indicou as amostras coletadas ao longo dos reservatórios, em contrapartida as bandas do vermelho $\mathrm{e}$ infravermelho apresentaram baixa respostas. Principalmente a turbidez teve melhores respostas com essas bandas no sensor MSI.

Esses resultados vão de encontro à pesquisa de Espinoza et al. (2012) que encontraram correlação entre os sólidos e essas bandas em águas com alta carga de sedimentos, ou seja, quanto maior a quantidade de sólidos maior a respostas nessas bandas. Mesmo assim, o sensor MSI conseguiu estabelecer uma correlação com a baixa quantidade de sólidos dos reservatórios analisados.

Os valores para a razão entre as bandas (NIR/RED e RED EDGE/RED) que apresentaram maiores valores de correlação $\left(R^{2}\right)$ estão apresentadas nas Tabelas 2 e 3. Em ambos os reservatórios os valores da razão das bandas NIR/RED do satélite LandSat 8 apresentaram melhores índices de correlação.

A correlação $\mathrm{R}^{2}$ foi utilizada em diferentes pesquisas como Robert et al., 2016 e Xie et al., 2018 e, segundo os autores, se apresenta como um fator explicativo capaz de modelar adequadamente a relação de variáveis ambientais e as reflectâncias das imagens.

Para o primeiro reservatório, os parâmetros de turbidez e sólidos totais nas bandas do Sentinel 2 e LandSat 8 (NIR/RED) apresentaram correlações a partir da quarta ordem polinomial, embora os índices do LandSat 8 foram maiores, ao contrário da etapa anterior.

Nos resultados da razão das bandas NIR/RED (Tabela 2), a análise polinomial de quarta ordem, para a turbidez apresentou índices $\mathrm{R}^{2}$ altos sendo $\mathrm{R}^{2}=0,855$ para as imagens do Sentinel 2A e $\mathrm{R}^{2}$ $=0,8507$ para o LandSat 8 .

Nesse mesmo grau a razão RED EDGE4/RED obteve $\mathrm{R}^{2}$ de 0,7422 e mesmo variando o grau do polinômio não foi possível aproximar de $\mathrm{R}^{2}=0,8$. Por outro lado, as bandas do LandSat 8 obtiveram alta correlação a partir da terceira ordem (Tabela 2).

Tabela 2 - valores de $\mathrm{R}^{2}$ das ordens polinomiais para os parâmetros de sólidos totais e turbidez com os resultados das razões de bandas no reservatório Amador Aguiar I.

\begin{tabular}{|c|c|c|c|}
\hline & \multicolumn{2}{|r|}{ Sentinel } & \multirow{2}{*}{$\begin{array}{l}\text { LandSat } 8 \\
\text { NIR/RED }\end{array}$} \\
\hline Ordem do polinômio & NIR/RED & RED EDGE4/RED & \\
\hline \multicolumn{4}{|c|}{ Turbidez } \\
\hline 2 & 0,5916 & 0,1343 & 0,3211 \\
\hline 3 & 0,7729 & 0,2238 & 0,5991 \\
\hline 4 & 0,855 & 0,289 & 0,8507 \\
\hline 5 & 0,896 & 0,7422 & 0,9505 \\
\hline 6 & 0,992 & 0,7774 & 0,9629 \\
\hline \multicolumn{4}{|c|}{ Sólidos totais } \\
\hline 2 & 0,177 & 0,4546 & 0,0206 \\
\hline 3 & 0,2422 & 0,7793 & 0,0655 \\
\hline 4 & 0,5884 & 0,7921 & 0,7548 \\
\hline 5 & $\mathbf{0 , 8 1 1 4}$ & 0,7924 & 0,9121 \\
\hline 6 & 0,9704 & 0,9337 & 0,9291 \\
\hline
\end{tabular}

Para o reservatório Amador Aguiar II observa-se que, de forma geral, os índices de correlação foram mais altos quando comparados ao outro reservatório (Tabela 3). Esses valores podem ser explicados pelos valores de sólidos totais e turbidez superiores encontrados no II indicando, inclusive, o carreamento parcial de sólidos de um reservatório para outro à jusante. A razão entre as bandas realçou os contrastes para identificação de sólidos totais, onde obtiveram índices melhores quando comparados com as bandas isoladamente. Contudo, a análise do diagrama de dispersão também apresentou índices melhores no reservatório Amador Aguiar II.

Robert et al. (2016) aplicaram o mesmo método que Espinoza et al. (2012) mas em reservatório que também possuem moderada carga de sedimentos e turbidez, e encontraram melhor correlação na razão das bandas NIR/RED.

No caso desta pesquisa, os valores baixos e sem variações significativas de sólidos e turbidez foi melhor representada pelos índices do que pelas bandas isoladas.

Os baixos valores de turbidez e sólidos totais demostram as características da região (geologia, profundidade, vegetação ciliar preservada e 
localização, pois são os últimos reservatórios de um sistema de cascata compostos por quatro reservatórios), as diferenças entre as amostras também foram pequenas o que indica uma homogeneidade na qualidade da água do complexo Capim Branco. Pode-se considerar dentro do padrão de normalidade o reservatório
Amador Aguiar II apresentar, mesmo que de forma sutil, maiores quantidades de sólidos totais e turbidez, pois, embora os dois reservatórios sejam utilizados para recreação, este último apresenta menos quantidade de vegetação ciliar, indicando que o uso e ocupação do solo das áreas do entorno influenciam na qualidade da água.

Tabela 3 - valores de $\mathrm{R}^{2}$ das ordens polinomiais para os parâmetros de turbidez e sólidos totais com os resultados das razões de bandas no reservatório Amador Aguiar II.

\begin{tabular}{c|c|c|c}
\hline & \multicolumn{2}{|c|}{ Sentinel } & LandSat 8 \\
\hline Ordem do polinômio & NIR/RED & RED EDGE4/RED & NIR/RED \\
\hline \multicolumn{4}{|c}{ Turbidez } \\
\hline $\mathbf{2}$ & 0,2815 & 0,031 & $\mathbf{0 , 8 6 1 6}$ \\
\hline $\mathbf{3}$ & 0,5856 & 0,1585 & $\mathbf{0 , 9 2 5 7}$ \\
\hline $\mathbf{4}$ & 0,5876 & 0,1591 & $\mathbf{0 , 9 8 5 5}$ \\
\hline $\mathbf{5}$ & 0,7786 & 0,5062 & $\mathbf{0 , 9 8 8}$ \\
\hline $\mathbf{6}$ & $\mathbf{0 , 9 7 7 9}$ & $\mathbf{0 , 8 4 9 9}$ & $\mathbf{0 , 9 9 4}$ \\
\hline \multicolumn{4}{|c|}{ Sólidos totais } \\
\hline $\mathbf{2}$ & 0,3421 & 0,0299 & $\mathbf{0 , 8 0 1 4}$ \\
\hline $\mathbf{3}$ & 0,5912 & 0,1167 & $\mathbf{0 , 9 1 5}$ \\
\hline $\mathbf{4}$ & $\mathbf{0 , 6 1 3 2}$ & 0,1411 & $\mathbf{0 , 9 1 8}$ \\
\hline $\mathbf{5}$ & $\mathbf{0 , 6 1 5 2}$ & 0,1842 & $\mathbf{0 , 9 1 8 1}$ \\
\hline 6 & 0,5792 & 0,2464 & 1,0183 \\
\hline
\end{tabular}

\section{CONCLUSÕES}

A análise dos pontos amostrados nos dois reservatórios apresentou, em geral, baixas quantidades de sólidos totais e turbidez, representando parâmetros físicos condizentes com a boa qualidade de água para os reservatórios analisados. No reservatório Amador Aguiar II, que apresentou sólidos totais e turbidez mais altos, a banda azul do satélite Sentinel 2, obteve refletância mais alta e a banda verde um pouco menor, fazendo com que a curva de refletância das duas bandas se aproximassem. No Amador Aguiar I a banda infravermelha teve os menores valores de refletância seguida da banda vermelha.

Esses resultados demonstram-se sensíveis à pequena presença desses parâmetros, o que em si é um resultado interessante do ponto de vista da aplicabilidade do método. Outro ponto de destaque refere-se ao uso das imagens do sensor MSI do satélite Sentinel 2A, especificamente as bandas azul e verde, que corroboraram as pesquisas referenciadas. Contudo, a variação dos sedimentos foi relacionada de forma mais intensa aos índices de razão utilizando as imagens do sensor OLI do Landsat8. Essas diferenças se devem, particularmente, à largura das bandas em cada faixa espectral dos dois sensores. Ainda assim, os valores de correlação com as imagens do OLI tiveram valores maiores e distribuídos desde polinômios de graus mais baixos. Isso demonstra consistência na resposta espectral e valores obtidos pela coleta de água. Dessa forma, existe uma potencial utilização de imagens orbitais gratuitas e de média resolução no monitoramento de turbidez e sólidos totais em reservatórios de médio porte, ou que tenham pequenas variações sazonais como o caso do cerrado brasileiro.

Como trabalhos futuros recomenda-se a aplicação de técnicas complementares como a fusão de imagens e outros satélites. Além disso, a viabilidade da técnica apresentada poderá ser confirmada por meio de uma série temporal mais ampla, sendo a coleta sistemática das amostras de água por longos períodos e a coincidência das aquisições das imagens com as datas das coletas a maior barreira para a completa validação de campo desta técnica.

\section{AGRADECIMENTOS}

O terceiro autor gostaria de agradecer o suporte do seguinte projeto: PQ/nível 2/CNPq $n^{\circ}$ 09/2018, proc. $n^{\circ} 310452 / 2018-0$. 


\section{REFERÊNCIAS}

AB'SABER, A. Os Domínios de Natureza no Brasil, Potencialidades paisagísticas. São Paulo: Atêlie Editorial. 4ª ed. 160 p., 2003.

ABDELMALIK, K.W. Role of statistical remote sensing for Inland water quality parameters prediction. The Egyptian Journal of Remote Sensing and Space Sciences, $N^{\circ} 21$, p. 193200. 2018.

ALCÂNTARA, E.; CURTARELLI, M.; KAMPEL, M.; STECH, J. Spatiotemporal total suspended matter estimation in Itumbiara reservoir with LandSat 8-8/OLI images. International Journal of Cartography, v. 2, n. 2, p. 14-165, 2016.

CONSÓRCIO CAPIM BRANCO DE ENERGIA - CCBE. 2005. Plano Diretor AHE's Capim Branco I e II. Disp. em $<$ http://www.ccbe.com.br/>. Acesso em: 16 nov. 2017.

DOMÍNGUEZ, J.A.; KUMHÁLOVÁ, J.; NOVÁK, P. Winter oilseed rape and winter wheat growth prediction using remote sensing methods. Plant Soil Environment, v. 61, n. 9: 410-416, 2015.

EL-ZEINY, A.E. \& EL-KAFRAWY, S. Assessment of water pollution induced by human activities in Burullus Lake using LandSat 8 operational land imager and GIS. The Egyptian Journal of Remote Sensing and Space Sciences. v. 20, p. 49-56. 2017.

ESPINOZA, V.R.; MARTINEZ, J.M.; LE TEXIER, M.; GUYOT, J.L.; FRAIZY, P.; MENESES, P.R. E OLIVEIRA, E.A. Study of sediment transport in the Madeira River, Brazil, using MODIS remote-sensing images. Journal of South American Earth Sciences, v 44, p. 4554, 2012.

JAFAR-SIDIK, M.; GOHIN, F.; BOWERS, D.; HOWARTH, J. \& HULL, T. The relationship between Suspended Particulate Matter and Turbidity at a mooring station in a coastal environment: consequences for satellitederived products. Oceanologia, v. 109, p. 365378, 2017.

NICHOL, J.E. \& NAZEER, M. Improved water quality retrieval by identifying optically unique water classes. Journal of Hydrology, v 541, p. 1119-1132, 2016.

NOVAIS, G.T., BRITO, J.L.S., SANCHES, F. DE O. Unidades Climáticas Do Triângulo
Mineiro/Alto Paranaíba. Revista Brasileira de Climatologia, v. 23, n. 14, 2018.

NOVO, E.; PRADO, M.L.M.; BARDY, R. Avaliação espaço-temporal da relação entre o estado trófico do reservatório de Barra Bonita (SP) e o potencial poluidor de sua bacia hidrográfica. Sociedade \& Natureza, v. 19, n. 2, p. 5-18, 2007.

PALMER, S.; KUTSER, T., HUNTER, P. Remote sensing of inland waters: challenges, progress and future directions. Remote Sensing of Environment, v. 157, p. 1-8, 2015. ROBERT, E.; GRIPPA, M.; KERGOAT, L.; PINET, S.; GAL, L.; COCHONNEAU, G.; MARTINEZ, J. Monitoring water turbidity and surface suspended sediment concentration of the Bagre Reservoir (Burkina Faso) using MODIS and field reflectance data. International Journal of Applied Earth Observation and Geoinformation, v. 52, p. 243-251, 2016.

ROUSE, J. W.; HAAS, R. H. \& SCHELL, J. A. Monitoring vegetation systems in the Great Plains with ERTS. In: ERTS-1 SYMPOSIUM,

3, Washington. Proceedings...Washington: NASA, v. 1, p. 309-317, 1974. Disp. em: https://ntrs.nasa.gov/archive/nasa/casi.ntrs.nas a.gov/1974002 2614.pdf

SERVIÇO DE ABASTECIMENTO DE SÃO PAULO - SABESP: Norma Técnica Interna NTS 019. São Paulo, 1999.

SOUZA C.H.W.; LAMPARELLI, R.A.C.; JUSTINA, D.D.; ROCHA, J.V. Estudo da Banda Red Edge do satélite Rapideye na discriminação da cobertura vegetal. In: SIMPÓSIO BRASILEIRO DE SENSORIAMENTO REMOTO, XVII, João Pessoa-PB, 2015. Anais...João Pessoa: INPE, 2015.

SOUZA, R.R.; COSTA R.A.; ASSUNÇÃO, H.F.; MELO, S.C. Variações pluviométricas no triangulo mineiro-mg. Geonordeste, Ano XX, n. 2, 2009.

SPITKÓ, T.; NAGY, Z.; ZSUBORI, Z.T.; SZÖKE， C.; BERZY， T.; PINTÉR， J.; MARTON, C.L. Connection between normalized difference vegetation index and yield in maize. Plant Soil Environ, v. 62, n. 7, 293-298, 2016,

WOŹNIAK, S.B.; DARECKI, M.; ZABŁOCKA, M.; BURSKA, D.; DERA, J. 
New simple statistical formulas for estimating surface concentrations of suspended particulate matter (SPM) and particulate organic carbon (POC) from remote-sensing reflectance in the southern Baltic Sea. Oceanologia, n. 3, p. 161 - 175, 2016.

XIE, Q.; DASH, J.; HUANG, W.; PENG, D.; QIN, Q.; MORTIMER, H.; CASA, R.; PIGNATTI, S.; LANEVE, G.; PASCUCCI, S.; DONG, Y.; YE, H. Vegetation Indices Combining the Red and Red-Edge Spectral Information for Leaf Area Index Retrieval. IEEE Journal of Selected Topics in Applied Earth Observations and Remote Sensing. 2018.
ZHANG, Y.; LI, Y.; SHI, K.; ZHU, G.; ZHOU, Y.; ZHANG, Y.; GUO, Y. Monitoring spatiotemporal variations in nutrients in a large drinking water reservoir and their relationships with hydrological and meteorological conditions based on LandSat 8 imagery. Science of the Total Enviroment, v. 1, n. 600, p. 1705-1717, 2017.

Submetido em 11 de março de 2020 Aceito para publicação em 15 de agosto de 2020 